\title{
Role of FN1 and GREB1 gene polymorphisms in endometriosis
}

\author{
CHAROULA MATALLIOTAKI ${ }^{1,2}$, MICHAIL MATALLIOTAKIS $^{1,2}$, NILUFER RAHMIOGLU ${ }^{3,4}$, \\ GEORGE MAVROMATIDIS $^{5}$, IOANNIS MATALLIOTAKIS ${ }^{2}$, GEORGE KOUMANTAKIS ${ }^{6}$, \\ KRINA ZONDERVAN ${ }^{3,4}$, DEMETRIOS A. SPANDIDOS ${ }^{7}$, GEORGE N. GOULIELMOS $^{8}$ and MARIA I. ZERVOU ${ }^{8}$ \\ ${ }^{1}$ Third Department of Obstetrics and Gynecology, Aristotle University of Thessaloniki, 54124 Thessaloniki; \\ ${ }^{2}$ Department of Obstetrics and Gynecology, Venizeleio and Pananio General Hospital of Heraklion, 71409 Heraklion, \\ Greece; ${ }^{3}$ Wellcome Trust Centre for Human Genetics, and ${ }^{4}$ Endometriosis CaRe Centre Oxford, \\ Nuffield Department of Women's and Reproductive Health, John Radcliffe Hospital, University of Oxford, \\ OX3 7BN Oxford, UK; ${ }^{5}$ Second Department of Obstetrics and Gynecology, Aristotle University of Thessaloniki, \\ 54124 Thessaloniki; ${ }^{6}$ Genesis Athens Clinic, 15232 Athens; ${ }^{7}$ Laboratory of Clinical Virology, \\ and ${ }^{8}$ Section of Molecular Pathology and Human Genetics, Department of Internal Medicine, \\ School of Medicine, University of Crete, 71003 Heraklion, Greece
}

Received March 1, 2019; Accepted April 16, 2019

DOI: $10.3892 / \mathrm{mmr} .2019 .10247$

\begin{abstract}
Endometriosis is a complex gynecological disorder, affecting up to $10 \%$ of women of childbearing age, characterized by the presence of functional endometrial tissue at ectopic positions generally within the peritoneum. It is a heritable condition influenced by multiple genetic, epigenetic and environmental factors, with an overall heritability estimated at approximately $50 \%$. The aim of the present study was to evaluate the association of rs1250248 and rs11674184 single nucleotide polymorphisms (SNPs), mapping to fibronectin 1 $(F N 1)$ and growth regulation by estrogen in breast cancer 1 (GREB1) genetic loci, respectively, with the risk of endometriosis. A total of 166 women with endometriosis (stages I-IV) who were hospitalized for the condition, diagnosed by laparoscopic intervention and histologically confirmed, and 168 normal controls were recruited and genotyped. Genotyping of the rs1250248 and rs11674184 SNPs was performed with TaqMan primer/probe sets. A significant association was detected with the A allele, as well as the AA and AG genotypes of rs1250248 (FNI) in patients with endometriosis, as well as in patients with stage I and II of the disease only. The rs11674184 SNP of the GREBI gene was not found to be associated with an increased susceptibility to endometriosis either for all patients (stages I-IV) or for subgroups of stage I and II or III and IV of the disease only. Our results demonstrated a
\end{abstract}

Correspondence to: Dr Maria I. Zervou, Section of Molecular Pathology and Human Genetics, Department of Internal Medicine, School of Medicine, University of Crete, 71003 Heraklion, Greece E-mail: mzervou@med.uoc.gr

Key words: endometriosis, genetic association, gene polymorphism, fibronectin 1, growth regulation by estrogen in breast cancer 1 genetic association between the rs1250248 (FN1) SNP and endometriosis at both the genotypic and allelic level. However, although rs11674184 of GREBl constitutes one of the most consistently associated SNPs with endometriosis in European ancestry populations, it was not found to be associated with endometriosis in this study.

\section{Introduction}

Endometriosis is a heritable, benign, chronic, estrogen-dependent, inflammatory, common gynecological disorder, affecting up to $10 \%$ of the female population during their reproductive years, representing a considerable cause of pain and infertility (1). Endometriosis is associated with chronic pelvic pain, dyspareunia, dysmenorrhea and infertility, and in women with this condition, the growth of endometrial tissue occurs in sites other than the uterine cavity, most commonly in the pelvic cavity, including the ovaries, the uterosacral ligaments, and the pouch of Douglas (2).

Endometriosis is characterized by a multifactorial pattern of inheritance, influenced by multiple genetic and environmental factors (3-5); however, the exact genomic basis of endometriosis remains unclear. Monozygotic twin-based and family studies have demonstrated an approximately $50 \%$ heritability for endometriosis $(6,7)$, while a strong familial aggregation has also been reported in a large pedigree of non-human primates (8). Previous linkage and candidate genetic studies on endometriosis did not succeed in identifying genetic variants strongly associated with risk of the disease (9), probably due to the small effect size for individual risk factors of the disease, which required studies based on large cohorts. Contrarily, genome-wide association studies (GWAS) and meta-analyses conducted in several series of samples have led to the identification of disease-susceptibility loci implicated in matrix remodeling, cell cycle regulation and signaling, cell adhesion, transcription regulation and inflammation, as well as immune, oxidative stress processes, as well as 
hormone receptors and metabolism (10-12). The list of novel endometriosis-associated loci is still being enriched due to novel GWAS and meta-analyses that are currently in progress. Importantly, these data only account for approximately 5\% of the disease variance (11). Of note, the role of epigenetics in the development of endometriosis, through interactions with environmental changes, has been investigated and the DNA methylation and histone post-translational modifications have been thoroughly investigated $(13,14)$.

Meta-analysis of European ancestry GWAS have identified 27 genome-wide significant loci associated with endometriosis (15). Several studies have genotyped key single nucleotide polymorphisms (SNPs) from GWAS to replicate the association in different samples and ethnic groups. Thus, the association of rs10859871 SNP, close to the Vezatin gene (VEZT) (16), CDKN2BAS rs1333049, rs7521902 close to Wnt family member 4 (WNT4), rs12700667 in an inter-genic region on 7p15.2 and fibronectin 1 (FN1) rs1250248 with endometriosis has been successfully replicated in an Italian ancestry population (16). Similarly, eight interleukin (IL)1A SNPs were successfully replicated in different ethnic populations (17). However, the chances of significant replication depend on the relative sample size in the replication and discovery samples.

To gain a better understanding of the genetic architecture of endometriosis in the Greek population, and encouraged by the successful replication of Wnt family member 4 (WNT4), VEZT and follicle stimulating hormone subunit beta (FSHB) gene association with endometriosis in this population (18), we sought to substantially replicate more data from recently conducted GWAS for endometriosis $(11,19)$. In particular, in the present study, we aimed to investigate whether rs1250248 and rs11674184 SNPs, mapping to fibronectin (FN1) and growth regulation by estrogen in breast cancer 1 (GREBl) gene loci, respectively, previously reported to be associated with endometriosis $(11,20)$, were associated with an increased risk of developing endometriosis and/or with progression to the minimal/mild (I/II) or moderate/severe stages (III-IV) of this condition in the Greek population. Furthermore, we attempted to detect any ethnic specific differences regarding the genetic association of these SNPs with endometriosis, considering that population differences for endometriosis have been reported previously in terms of genetic susceptibility and disease manifestations in Greek women with endometriosis $(18,21)$.

\section{Materials and methods}

Patient population and study design. In this case control association study, 334 women were enrolled (166 endometriosis patients and 168 controls) from the Department of Obstetrics and Gynecology of Venizeleion Hospital of Heraklion. The average age of the Greek endometriosis and control cohorts was $32.25 \pm 7.1$ and $29.49 \pm 6.7$ years, respectively. All women with endometriosis were diagnosed surgically (laparotomy or laparoscopy), and the disease was confirmed histologically from biopsies. The staging of the disease was performed according to the revised American Fertility Society classification (22) and is depicted in Table I. All members of the control group had given birth to 2 to $5(2.3 \pm 0.6)$ children and had no previous medical record of chronic pelvic pain, dysmenorrhea, or dyspareunia. Both the cases and controls were unrelated, living in the same
Table I. Patients characteristics.

\begin{tabular}{ll}
\hline $\begin{array}{l}\text { Age (years) } \\
(\text { mean } \pm \text { SD) }\end{array}$ & $\begin{array}{c}\text { Patients with endometriosis } \\
\text { - stage of endometriosis }\end{array}$ \\
\hline $29.49 \pm 6.7$ & $36(21.69 \%)-$ I \\
& $51(30.72 \%)-$ II \\
& $36(21.69 \%)-$ III \\
& $43(25.90 \%)-$ IV \\
& $87(52.41 \%)-$ I+II \\
& $79(47.59 \%)-$ III+IV
\end{tabular}

urban environment and originated from the same Greek population. All subjects were of self-reported Greek origin. This study was performed at the Section of Molecular Pathology and Human Genetics of the Medical School of Crete and was carried out in compliance with the declaration of Helsinki. The Ethics Committee of Venizeleio General Hospital of (ECHR no. 47/773) approved the overall study and written informed consent was obtained from all the patients. The medical records were collected by the clinicians and pathologists, including surgical procedures and findings.

Genotyping. Whole blood was collected pre-operatively in ethylenediaminetetraacetic acid (EDTA)-containing tubes. Genomic DNA was isolated from peripheral blood leukocytes using a commercial kit (PureLink ${ }^{\circledR}$ Genomic DNA Mini kit; Invitrogen; Thermo Fisher Scientific) according to the manufacturer's instructions. The extracted DNA was stored at $-20^{\circ} \mathrm{C}$ until analyzed. The allelic discrimination of the FN1 and GREB1 gene SNPs, rs1250248 and rs11674184, respectively, was carried out using pre-made TaqMan SNP genotyping assays from Applied Biosystems, according to the Applied Biosystems protocol (catalogue ID: C_2110731_10 and C_7728566_10 for rs1250248 and rs11674184, respectively), as previously described in detail by Matalliotakis et al (18). Allelic discrimination plots were all reviewed individually for quality. Each assay was run with negative controls. For quality control, a random $10 \%$ of the samples were amplified twice to ensure accuracy of the results, with the reproducibility rate being $100 \%$. The genotyping success for all the SNPs analyzed was $>98 \%$.

Statistical analysis. All the cases and controls used in the analysis were unrelated. Statistical analysis was performed using the GraphPad Prism statistical program (GraphPad Software), by applying the additive model. The Chi-squared $\left(\chi^{2}\right)$ test, with one or two degrees of freedom or Fisher's exact test was used to examine differences of genotype and allele frequencies between patients and controls, where all SNPs had a call rate of $>98 \%$. A two-tailed P-value $<0.05$ was defined as statistically significant. Odds ratios (OR) and $95 \%$ confidence intervals (CI) were calculated. The genetic variants under investigation were evaluated for deviation from the Hardy-Weinberg equilibrium (HWE) by comparing observed and expected genotype frequencies by means of Chi-squared $\left(\chi^{2}\right)$ test or Fisher's exact test in the control groups (by using the program named 'Calculate'; Copyright TRG, SR, INMD, 2008). The distribu- 
Table II. Genotypes and allele frequencies of the FN1 rs1250248 polymorphism analyzed in 166 women with endometriosis and 168 healthy controls.

\begin{tabular}{|c|c|c|c|c|}
\hline Genotypes/Alleles & Endometriosis & Controls & P-value & OR $(95 \% \mathrm{CI})$ \\
\hline Genotypes & $n=166$ & $\mathrm{n}=168$ & & \\
\hline GG & $69(41.57 \%)$ & $102(60.71 \%)$ & & \\
\hline $\mathrm{AG}$ & $69(41.57 \%)$ & $50(29.76 \%)$ & 0.0041 & $2.04(1.27-3.28)$ \\
\hline AA & $28(16.86 \%)$ & $16(9.52 \%)$ & 0.0066 & $2.59(1.30-3.14)$ \\
\hline Alleles & $\mathrm{n}=332$ & $\mathrm{n}=336$ & & \\
\hline $\mathrm{G}$ & $207(62.35 \%)$ & $254(75.59 \%)$ & & \\
\hline A & $125(37.65 \%)$ & $82(24.41 \%)$ & 0.0020 & $1.87(1.34-2.61)$ \\
\hline
\end{tabular}

OR, odds ratio; CI, confidence interval. Values in bold font indicate statistically significant differences $(\mathrm{P}<0.05)$.

Table III. Genotype and allele frequency of the FN1 rs1250248 polymorphism analyzed in 87 women with endometriosis (stage I and II) and 168 healthy controls.

\begin{tabular}{|c|c|c|c|c|}
\hline Genotypes/Alleles & Endometriosis & Controls & P-value & OR $(95 \% \mathrm{CI})$ \\
\hline Genotypes & $\mathrm{n}=87$ & $n=168$ & & \\
\hline GG & $30(34.48 \%)$ & $102(60.71 \%)$ & & \\
\hline $\mathrm{AG}$ & $37(42.53 \%)$ & $50(29.76 \%)$ & 0.0026 & $2.51(1.40-4.53)$ \\
\hline AA & $20(22.99 \%)$ & $16(9.52 \%)$ & 0.0003 & 4.25 (1.96-9.21) \\
\hline Alleles & $\mathrm{n}=174$ & $n=336$ & & \\
\hline $\mathrm{G}$ & $97(55.75 \%)$ & $254(75.59 \%)$ & & \\
\hline A & $77(44.25 \%)$ & $82(24.41 \%)$ & $<0.0001$ & $2.45(1.66-3.62)$ \\
\hline
\end{tabular}

OR, odds ratio; CI, confidence interval. Values in bold font indicate statistically significant differences $(\mathrm{P}<0.05)$.

tion of genotypes in case group for all 2 SNPs examined were found to be under HWE $(\mathrm{P}>0.01)$.

\section{Results}

rs1250248 SNP of FN1. In the case of the intronic rs1250248 SNP located in the FN1 gene, a statistically significant difference was found in the frequency of the A allele in the genotypes and controls $(\mathrm{P}=0.0020, \mathrm{OR}=1.87,95 \%$ CI 1.34-2.61) (Table II). Additionally, a statistically significant difference was observed in the frequencies of the AA and AG genotypes in cases vs. controls $(\mathrm{P}=0.0066, \mathrm{OR}=2.59,95 \% \mathrm{CI}, 1.30-3.14$ and $\mathrm{P}=0.0041$, $\mathrm{OR}=2.04,95 \% \mathrm{CI}, 1.27-3.28$, respectively) (Table II).

Of note, in an analysis conducted by endometriosis stage and apart from the small sample size for each stage, a significant association was detected regarding the AA and AG genotypes of this SNP in patients with stage I/II of the disease and the controls $(\mathrm{P}=0.0003, \mathrm{OR}=4.25,95 \% \mathrm{CI}, 1.96-9.21$ and $\mathrm{P}=0.0026$, $\mathrm{OR}=2.51,95 \% \mathrm{CI}, 1.40-4.53$, respectively), as depicted in Table III. Furthermore, evidence for the association with endometriosis cases stratified to stages I and II was found for the A allele $(\mathrm{P}<0.0001, \mathrm{OR}=2.45,95 \%$ CI 1.66-3.62) (Table III). Notably, no evidence for the association with endometriosis was observed either at the genotypic or allelic level when patients with endometriosis of stages III/IV were analyzed (Table IV), which differs from previous findings in other ethnic populations (23). rs11674184 SNP of GREB1. In the case of the rs11674184 SNP of the GREBl gene, patients with endometriosis did not present any increase in genotype or allele frequencies compared with the controls $(\mathrm{P}=0.31$, OR $1.18,95 \%$ CI $0.86-1.59$ for the $\mathrm{G}$ allele; and $\mathrm{P}=0.36$, OR $1.37,95 \%$ CI $0.75-2.48$ and $\mathrm{P}=0.48$, OR 1.24, 95\% CI 0.71-2.16 for the GG and TG genotypes, respectively) (Table V), apart from findings reported in other studies, showing that allele $\mathrm{G}$ of the rs11674184 is a risk allele for endometriosis (24). Similarly, when patients were analyzed according to the severity of the disease, no significant association with endometriosis was detected in patients with stage I/II or III/IV of the disease either at the genotype or allele frequencies of rs11674184. Thus, when the allele ' $G$ ' frequencies of patients with endometriosis of stage I/II or III/IV were compared with the controls, no statistically significant differences were observed $(\mathrm{P}=0.92$, OR $1.03,95 \% \mathrm{CI} 0.71-1.48$ and $\mathrm{P}=0.12$, OR $1.36,95 \%$ CI $0.92-2.00$ for stages I/II and III/IV, respectively) (detailed genotype data not shown).

\section{Discussion}

Nowadays, a better understanding of the genetic risk factors associated with endometriosis has been achieved by using advanced technological applications, including GWAS and next generation sequencing (NGS). However, accumulated data point out the existence of a number of differences in genetic 
Table IV. Genotype and allele frequency of the FN1 rs1250248 polymorphism analyzed in 87 women with endometriosis (stage III and IV) and 168 healthy controls.

\begin{tabular}{|c|c|c|c|c|}
\hline Genotypes/Alleles & Endometriosis & Controls & P-value & OR $(95 \% \mathrm{CI})$ \\
\hline Genotypes & $\mathrm{n}=79$ & $\mathrm{n}=168$ & & \\
\hline $\mathrm{GG}$ & $39(49.37 \%)$ & $102(60.71 \%)$ & & \\
\hline $\mathrm{AG}$ & $32(40.51 \%)$ & $50(29.76 \%)$ & 0.10 & $1.67(0.94-2.98)$ \\
\hline AA & $8(10.13 \%)$ & $16(9.52 \%)$ & 0.62 & $1.31(0.52-3.30)$ \\
\hline Alleles & $\mathrm{n}=158$ & $n=336$ & & \\
\hline $\mathrm{G}$ & $110(69.62 \%)$ & $254(75.59 \%)$ & & \\
\hline A & $48(30.38 \%)$ & $82(24.41 \%)$ & 0.19 & $1.35(0.88-2.05)$ \\
\hline
\end{tabular}

OR, odds ratio; CI, confidence interval.

Table V. Genotypes and alleles frequency of the GREB1 rs11674184 polymorphism analyzed in 165 women with endometriosis and 168 healthy controls.

\begin{tabular}{|c|c|c|c|c|}
\hline Genotypes/Alleles & Endometriosis & Controls & P-value & OR $(95 \%$ CI $)$ \\
\hline Genotypes & $\mathrm{n}=165$ & $\mathrm{n}=168$ & & \\
\hline $\mathrm{TT}$ & $55(33.33 \%)$ & $50(29.76 \%)$ & & \\
\hline TG & $77(46.67 \%)$ & $77(45.83 \%)$ & 0.48 & $1.24(0.71-2.16)$ \\
\hline GG & $33(20.00 \%)$ & $41(24.41 \%)$ & 0.36 & $1.37(0.75-2.48)$ \\
\hline Alleles & $\mathrm{n}=330$ & $\mathrm{n}=336$ & & \\
\hline $\mathrm{T}$ & $187(56.67 \%)$ & $177(52.68 \%)$ & & \\
\hline $\mathrm{G}$ & $143(43.33 \%)$ & $159(47.32 \%)$ & 0.31 & $1.18(0.86-1.59)$ \\
\hline
\end{tabular}

OR, odds ratio; CI, confidence interval.

associations with the disease across different world populations (24) and, therefore, it is evident that replication studies in multiple populations have to be conducted in order for the genetic basis of endometriosis to be clarified.

In the present study, we identified that the rs1250248 SNP was significantly associated with endometriosis in the Greek population in 'all endometriosis' cases, as well as in the subgroup of stages I and II. Notably, previous studies have demonstrated a genome-wide significant association with the rs1250248 SNP of FN1 locus, particularly with moderate-to-severe endometriosis $(20,25)$. A meta-analysis based on four independent GWAS and four replication datasets, confirmed consistency of effects across datasets and populations for rs1250248 in FN1, providing evidence close to the genome-wide significance threshold of $\mathrm{P}<5 \times 10^{-8}(26)$. This SNP is located in an intronic region between exon 10 and exon 11 in $F N 1$ and, in particular, on a transcription factor-binding site according to ENCODE (27). FN1 is a glycoprotein of the extracellular matrix, which is also present in plasma, and at the cell surface. It mediates various cellular interactions with the extracellular matrix and has important roles in cell adhesion and migration processes, host defense, would healing, growth, differentiation and metastasis $(28,29)$. Furthermore, it has been shown that $S O X 2$, encoding a transcription factor that targets FN1, is a key gene regulating cell migration in ovarian cancer (30). FN1 is involved in the sex steroid hormone pathway and in a recent study, we demonstrated the replication of rs11031006, associated with endometriosis in the FSHB gene, which is also involved in the regulation of the sex steroid hormone pathway in the Greek population (18). However, the effects of the variants in underlying biological pathways need to be determined (25). Taking into consideration that rs1250248 SNP is located within the FN1 gene, it can be assumed that its biological consequences may act through this gene. However, functional studies are required to better understand its role in endometriosis disease etiology. These studies will aid towards the understanding of how the confirmed gene variant is involved in the disease phenotype, including gene expression and protein levels in different tissues, relevant to endometriosis, such as the eutopic and ectopic endometrium.

It is worth noting that the number of the loci identified by GWAS is increasing as the proportion of cases analyzed are limited to more severe disease stages (stage III/IV endometriosis) rather than stage $\mathrm{I} / \mathrm{II}$, thus indicating that moderate to severe endometriosis cases have greater genetic burden relative to minimal or mild disease (25). However, as regards the rs1250248 FN1 SNP, in the present study, the milder disease stages (stage I/II endometriosis) were found to be associated with endometriosis rather than the more severe stages (III/ IV). Evidently, detailed surgical data from the cases will confer to a better assessment of the genetic variants associated with different stages of the disease. In an attempt to develop new options dealing with the better treatment and the therapy of women with endometriosis, focusing on subtypes of the diseases, it is necessary to understand the fundamental 
pathogenic mechanisms underlying the disease and gain novel insights from the risk gene loci detected through genetic studies. To this direction, the small effects of various identified SNPs should not to be underestimated, given that their targets may have a remarkable therapeutic value (31).

Previous research has identified genome-wide, endometriosis-associated significant variants, namely rs11674184 and rs13394619 in GREB1 on 2p25.1 (24). However, the present study did not reveal any association of the rs11674184 SNP investigated in the Greek population. The analyzed rs11674184 SNP has a minor allele frequency (MAF) of 0.47 in the Greek population; however, this SNP has a MAF of 0.61 in Australians, as reported by Fung et al (32). Notably, the same SNP was found to have a MAF of 0.61 in a previous meta-analysis (11). GREBl is an estrogen-regulated gene that was first identified in breast cancer cell lines and tumors (33). Its increased expression in peritoneal eutopic endom triotic lesions probably reflects a potential role in estrogen-dependent growth of endometriosis (34). Nevertheless, the underlying biological mechanism through which GREB1 plays a role in estrogen-stimulated cell proliferation in endometriosis remains still elusive.

The rs1250248 of FNl has a MAF of 0.24 in the Greek population, as observed in the present study (Table II). Notably, this SNP had a MAF of 0.29 in a meta-analysis conducted by Sapkota et al (11), 0.33 in an Icelandic population (11) and 0.21 in a metaanalysis performed by Rahmioglu et al (25). The current study has some limitations, considering that only the single lead SNPs from the FN1 and GREB1 genes were genotyped. However, these lead SNPs are not necessarily the causal variants, but they 'tag' the causal SNPs. Fine-mapping studies are required in order to identify the disease-causing SNPs. Apparently, the failure to replicate previous results regarding the rs11674184 SNP of GREB1 does not eliminate the fact that there could be other genetic variants in/ around the GREBl locus that are associated with endometriosis in the Greek population. Hence, better coverage of the common variation in/around the reported by GWAS endometriosispredisposing SNPs is required in the Greek population. Notably, given that the replication Greek sample was smaller than the (original) discovery sample, the lack of significant replication does not constitute a real failure to replicate the original finding. Furthermore, genotyping in large case-control groups is necessary for the identification of more robust findings.

In conclusion, this study demonstrated that the FN1 but not the GREB1 polymorphisms analyzed may be involved in the development of endometriosis in Greek women and emphasize the importance of assessing genetic variants in different populations, even within Europe, aiming to define the genetic architecture of endometriosis. Moreover, further investigations are required to clarify the possible role of the FN1 SNP in the clinical course of endometriosis and elucidate the mechanism through which rs1250248 increases the risk of developing this disease.

\section{Acknowledgements}

The authors would like to thank all the clinicians and the pathologists for providing the data and pathology reports in this study.

\section{Funding}

No funding was received.

\section{Availability of data and materials}

The datasets used and/or analyzed during the current study are available from the corresponding author on reasonable request.

\section{Authors' contributions}

CM, NR, KZ, MM, GNG and MIZ conceived and designed the study. MM, CM, GM, GK and IM obtained the data. DAS, NR, MIZ, GM, GNG and CM analyzed and interpreted the data. CM, MIZ, GK and IM drafted the manuscript. MM, GNG, NR, DAS, GM and KZ critically revised the manuscript. All authors have read and approved the manuscript.

\section{Ethics approval and consent to participate}

The Ethics Committee for Human Research of Venizeleio Hospital approved the respective protocol (ECHR no. 47/773). Informed consent was obtained from all the participants.

\section{Patient consent for publication}

Not applicable.

\section{Competing interests}

$\mathrm{KZ}$ has scientific collaborations in the area of endometriosis with Bayer AG (Leverkusen, Germany), Roche Diagnostics (Basel, Switzerland) and MDNA.DAS is the Editor-in-Chief for the journal, but had no personal involvement in the reviewing process, or any influence in terms of adjudicating on the final decision, for this article. The other authors declare that they have no competing interests.

\section{References}

1. Burney RO and Giudice LC: Pathogenesis and pathophysiology of endometriosis. Fertil Steril 98: 511 519, 2012.

2. Halis G and Arici A: Endometriosis and inflammation in infertility. Ann N Y Acad Sci 1034: 300-315, 2004.

3. Simpson JL, Elias S, Malinak LR and Buttram VC Jr: Heritable aspects of endometriosis. I. Genetic studies. Am J Obstet Gynecol 137: 327-331, 1980.

4. Coxhead D and Thomas EJ: Familial inheritance of endometriosis in a British population. A case control study. J Obstet Gynaecol 13: 42-44, 1993.

5. Stefansson H, Geirsson RT, Steinthorsdottir V, Jonsson H, Manolescu A, Kong A, Ingadottir G, Gulcher J and Stefansson K: Genetic factors contribute to the risk of developing endometriosis. Hum Reprod 17: 555-559, 2002.

6. Treloar SA, O'Connor DT, O'Connor VM and Martin NG: Genetic influences on endometriosis in an Australian twin sample.sueT@qimr.edu.au. Fertil Steril 71: 701-710, 1999.

7. Saha R, Pettersson HJ, Svedberg P, Olovsson M, Bergqvist A, Marions L, Tornvall P and Kuja-Halkola R: Heritability of endometriosis. Fertil Steril 104: 947-952, 2015.

8. Zondervan KT, Weeks DE, Colman R, Cardon LR, Hadfield R, Schleffler J, Trainor AG, Coe CL, Kemnitz JW and Kennedy SH: Familial aggregation of endometriosis in a large pedigree of rhesus macaques. Hum Reprod 19: 448-455, 2004.

9. Montgomery GW, Nyholt DR, Zhao ZZ, Treloar SA, Painter JN, Missmer SA, Kennedy SH and Zondervan KT: The search for genes contributing to endometriosis risk. Hum Reprod Update 14: 447-457, 2008.

10. Kobayashi $H$,Imanaka $S, N a k a m u r a ~ H$ and Tsuji A: Understanding the role of epigenomic, genomic and genetic alterations in the development of endometriosis (Review). Mol Med Rep 9: 1483-1505, 2014 
11. Sapkota Y, Steinthorsdottir V, Morris AP, Fassbender A, Rahmioglu N, De Vivo I, Buring JE, Zhang F, Edwards TL, Jones S, et al; iPSYCH-SSI-Broad Group: Meta-analysis identifies five novel loci associated with endometriosis highlighting key genes involved in hormone metabolism. Nat Commun 8 : 15539, 2017.

12. UImari O, Rahmioglu N, Nyholt D, Vincent K, Missmer S, Becker C, Morris AP, Montgomery GW and Zondervan K: Genome-wide genetic analyses highlight MAPK signalling in the oathogenesis of endometriosis. Hum Reprod 32: 780-793, 2017.

13. Wang Z, Zang C, Rosenfeld JA, Schones DE, Barski A, Cuddapah S, Cui K, Roh TY, Peng W, Zhang MQ, et al: Combinatorial patterns of histone acetylations and methylations in the human genome. Nat Genet 40: 897-903, 2008

14. Tan M, Luo H, Lee S, Jin F, Yang JS, Montellier E, Buchou T, Cheng Z, Rousseaux S, Rajagopal N, et al: Identification of 67 histone marks and histone lysine crotonylation as a new type of histone modification. Cell 146: 1016-1028, 2011.

15. Rahmioglu N, Banasik, Christofidou P, Danning R, Galarneau G Giri A, MacGregor S, Mortlock S, Sapkota, Y, Schork JA, et al: Large-scale genome-wide association meta-analysis of endometriosis reveals 13 novel loci and genetically-associated comorbidity with other pain conditions. bioRxiv preprint first posted online Sept 7, 2018; doi: http://dx.doi.org/10.1101/406967.

16. Pagliardini L1, Gentilini D, Vigano' P, Panina-Bordignon P, Busacca M, Candiani M and Di Blasio AM: An Italian association study and meta-analysis with previous GWAS confirm WNT4, CDKN2BAS and FN1 as the first identified susceptibility loci for endometriosis. J Med Genet 50: 43-46, 2013.

17. Sapkota Y, Low SK, Attia J, Gordon SD, Henders AK, Holliday EG, MacGregor S, Martin NG, McEvoy M, Morris AP, et al: Association between endometriosis and the interleukin 1A (IL1A) locus. Hum Reprod. 30: 239-248, 2015.

18. Matalliotakis M, Zervou MI, Matalliotaki C, Rahmioglu N, Koumantakis G, Kalogiannidis I, Prapas I, Zondervan K, Spandidos DA, Matalliotakis I, et al: The role of gene polymorphisms in endometriosis. Mol Med Rep 16: 5881-5886, 2017.

19. Sapkota Y, Fassbender A, Bowdler L, Fung JN, Peterse D, O D, Montgomery GW, Nyholt DR and D'Hooghe TM: Independent replication and meta-analysis for endometriosis risk loci. Twin Res Hum Genet 18: 518-525, 2015.

20. Zondervan KT, Rahmioglu N, Morris AP, Nyholt DR, Montgomery GW, Becker CM and Missmer SA: Beyond endometriosis genome-wide association study: From genomics to phenomics to the patient. Semin Reprod Med 34: 242-254, 2016.

21. Matalliotakis M, Zervou MI, Eliopoulos E, Matalliotaki C, Rahmioglu N, Kalogiannidis I, Zondervan K, Spandidos DA, Matalliotakis I and Goulielmos GN: The role of IL 16 gene polymorphisms in endometriosis. Int J Mol Med 41: 1469-1476, 2018

22. The American Fertility Society: Revised American Fertility Society classification of endometriosis: 1985 . Fertil Steril 43: $351-352,1985$.
23. Rahmioglu N, Montgomery GW and Zondervan KT: Genetics of endometriosis. Wom Health Lond 11: 577-586, 2015.

24. Nyholt DR, Low SK, Anderson CA, Painter JN, Uno S, Morris AP, MacGregor S, Gordon SD, Henders AK, Martin NG, et al: Genome-wide association meta-analysis identifies new endometriosis risk loci. Nat Genet 44: 1355-1359, 2012.

25. Rahmioglu N, Nyholt DR, Morris AP, Missmer SA, Montgomery GW and Zondervan KT: Genetic variants underlying risk of endometriosis: Insights from meta-analysis of eight genome-wide association and replication datasets. Hum Reprod Update 20: 702-716, 2014.

26. Painter JN, Anderson CA, Nyholt DR, Macgregor S, Lin J, Lee SH, Lambert A, Zhao ZZ, Roseman F, Guo Q, et al: Genome-wide association study identifies a locus at $7 \mathrm{p} 15.2$ associated with the development of endometriosis. Nat Genet 43: 51-54, 2011.

27. ENCODE Project Consortium: An integrated encyclopedia of DNA elements in the human genome. Nature 489: 57-74, 2012.

28. Pankov R and Yamada KM: Fibronectin at a glance. J Cell Sci 115: 3861-3863, 2002.

29. Soikkeli J, Podlasz P, Yin M, Nummela P, Jahkola T, Virolainen S, Krogerus L, Heikkilä P, von Smitten K, Saksela O and Hölttä E: Metastatic outgrowth encompasses COL-I, FN1, and POSTN up-regulation and assembly to fibrillar networks regulating cell adhesion, migration, and growth. Am J Pathol 177: 387-403, 2010.

30. Lou X, Han X, Jin C, Tian W, Yu W, Ding D, Cheng L, Huang $\mathrm{B}$, Jiang $\mathrm{H}$ and Lin B: SOX2 targets fibronectin 1 to promote cell migration and invasion in ovarian cancer: new molecular leads for therapeutic intervention. OMICS 17: 510-518, 2013.

31. Barrett JC, Dunham I and Birney E: Using human genetics to make new medicines. Nat Rev Genet 16: 561-562, 2015.

32. Fung JN, Holdsworth-Carson SJ, Sapkota Y, Zhao ZZ, Jones L, Girling JE, Paiva P, Healey M, Nyholt DR, Rogers PA and Montgomery GW: Functional evaluation of genetic variants associated with endometriosis near GREB1. Hum Reprod 30: 1263-1275, 2015

33. Ghosh MG, Thompson DA and Weigel RJ: PDZK1 and GREB1 are estrogen-regulated genes expressed in hormone-responsive breast cancer. Cancer Res. 60: 6367-6375, 2000.

34. Pellegrini C, Gori I, Achtari C, Hornung D, Chardonnens E, Wunder D, Fiche $M$ and Canny GO: The expression of estrogen receptors as well as GREB1, c-MYC, and cyclin D1, estrogen-regulated genes implicated in proliferation, is increased in peritoneal endometriosis. Fertil Steril 98: 1200-1208, 2012.

This work is licensed under a Creative Commons Attribution-NonCommercial-NoDerivatives 4.0 International (CC BY-NC-ND 4.0) License. 